\title{
Robust Brain-computer interface for virtual Keyboard (RoBIK): project results
}

Louis Mayaud (1-4), Sabine Filipe (5), Lucie Pétégnief (6), Olivier Rochecouste (7) and Marco Congedo (8).

\section{Authors' affiliations:}

(1) INSERM, Centre d'Investigation Clinique et d'Innovation technologique (CICIT), UMR805, Garches, France

(2) EA4497, Université Versailles Saint-Quentin (UVSQ), Versailles, France

(3) Hôpital Raymond Poincaré, APHP, Garches, France

(4) Institute of Biomedical Engineering (IBME), University of Oxford, Oxford, UK

(5) CEA/LETI, MINATEC Campus, Department DTBS, Grenoble, France

(6) DIXI Microtechniques Medical, Besançon, France

(7) Davidson Consulting, Rennes, France

(7) GIPSA-Lab, University of Grenoble and CNRS, Grenoble, France

\section{Corresponding author:}

Louis Mayaud

CICIT, Hôpital Raymond Poincaré

104, Boulevard raymond Poincaré

92380 Garches

Email: louis.mayaud@gmail.com

Tel: +33650663491 


\begin{abstract}
Brain-Computer Interface $(\mathrm{BCl})$ is a technology that translates the brain electrical activity into a command for a device such as a robotic arm, a wheelchair or a spelling device. $\mathrm{BCls}$ have long been described as an assistive technology for severely disabled patients because they completely bypass the need for muscular activity. The clinical reality is however dramatically different and most patients who use $\mathrm{BCls}$ today are doing so as part of constraining clinical trials. To achieve the technological transfer from bench to bedside, $\mathrm{BCl}$ must gain ease of use and robustness of both measure (Electroencephalography, EEG) and interface (signal processing and applications).

The RoBIK project (Robust Brain-computer Interface for virtual Keyboard) aimed at the development of a $\mathrm{BCl}$ system for communication that could be used on a daily basis by patients without the help of a trained team of researchers. To guide further developments clinicians first assessed patients' needs. The prototype subsequently developed consisted in a 14 felt-pad electrodes EEG headset sampling at $256 \mathrm{~Hz}$ by an electronic component capable of transmitting signals wirelessly. The application was a virtual keyboard generating a novel stimulation paradigm to elicit P300 Evoked Related Potentials (ERPs) for communication. Raw EEG signals were treated with OpenViBE opensource software including novel signal processing and stimulation techniques.
\end{abstract}

\title{
Keywords
}

Brain-Computer Interface; Assistive Technology; Electro-encephalography; 


\section{Introduction}

Brain-Computer Interfaces $(\mathrm{BCl})$ is an old technology [1] that translates the brain electrical activity into a command for a device [2]. As illustrated in Figure 1, BCls are usually characterized by: (1) an EEG recording modality, (2) a paradigm that relates stimulations or neurofeedback to some specific brain activity and (3) an interface usually connecting an application by the mean of pattern recognition techniques.

In practice, $\mathrm{BCl}$ can be achieved with any modality recording the brain's activity, the choice of which is driven by the desired trade-off between performance and the risks associated with the technique: typically, the more invasive the technique, the higher the performance and the lower its safety [3]. For instance, microarray electrodes, which are directly connected to neurons, offer unequalled capabilities [4] but are associated with important risks, in addition to a relatively short lifetime of a few months [3]. Electrocorticography (ECoG) $[5,6]$ records signals below the scalp without penetrating the dura mera, which offers an interesting balance between performance and risks even though this still requires a major surgical procedure. Non invasive modalities are more widely used and consist of a large variety of techniques such as functional Magnetic Resonance Imagery (fMRI) [79], Magneto-EncephaloGraphy (MEG) [10, 11], Near Infrared Spectroscopy (NIRS) [12, 13], or Electroencephalography (EEG) [14-17].

EEG based $\mathrm{BCls}$ have gained great popularity amongst researchers thanks to its convenience and the dramatic drop in price that resulted in the recent progress in electronic components mass production. However, despite its widespread use, EEG modality has seen very little improvements 
over the past two decades and still suffers from major drawbacks [18]: electrode setup is a tricky and time-consuming process, which is prohibitive to daily use by patients. In particular caretakers do not necessarily have the time or the skill to use current EEG systems. Attempts to address such issues includes the use of caps with pre-positioned gel-based [19] or dry [20-22] electrodes potentially interfaced with active systems [23-25]. More recently, the Emotiv headset [26-28] has open the path to low cost EEG for public that could successfully be used in $\mathrm{BCl}$ designs even though its performance was proven to be overall lower than traditional systems [29-31].

$\mathrm{BCl}$ paradigms are numerous and are typically divided into synchronous or asynchronous ones depending on whether the speed of the system is fixed (and related to the stimulation pace) or not. An asynchronous paradigm, like the motor rhythms [32-34], usually requires a few neurofeedback training sessions (over a few days) for the subject to control the EEG feature that will be used to control the interface. The system speed will then solely be related to the user's ability to control his cerebral pattern leaving room for progress and finally offering an intuitive interface. A synchronous paradigm on the other hand exploits subject cerebral response to various types of stimulations. The response can be exogenous (that is directly related to the stimulus characteristics) like in Steady State Visual Evoked Potentials (SSVEP) $[35,36]$ or endogenous (related to the subject's cognitive activity) like in "P300" designs. A P300 is a positive Event Related Potential (ERP) occurring about 300 ms after the presentation of a "rare" stimulus [37], which has been broadly used in different type of $\mathrm{BCl}$ paradigm, mainly visual $[38,39]$ and auditory ones [40]. Synchronous paradigms usually require shorter training period (typically a few minutes) during which algorithms will try to optimize recognition of the subject's EEG features. 
In most $\mathrm{BCl}$ applications, stimulations, signal pre-processing [41] and pattern recognition algorithms [42] are handled by the same platform $[43,44]$. Identification of specific EEG features finally allows control of a device or an application without the need of any sort of muscular activity. Thanks to this specificity and possibly because of the relatively slow throughput of these systems, applications have so far concentrated on restoration of control and communication in severely disabled patients [45]. Non-invasive $\mathrm{BCl}$ s have indeed successfully been used to control wheelchairs [46, 47], a robotic arm [48], computer applications [49] and spelling devices such as the broadly used "P300 speller" [39, 50]. Despite recent effort of the scientific community, the deployment of these applications still requires the skill of highly trained and experienced staff.

Indeed, the tremendous scientific and medical literature hides a dramatically different clinical reality: most patients using this technology nowadays are included in clinical trials [50-54] and therefore benefit from an important support from medical and technical staffs. To date, it seems very unlikely for a patient's family at home, or a medical staff in a rehabilitation or an intensive care unit, to have both the availability and the skill to install an EEG system and therefore run a $\mathrm{BCl}$ application. To complete the technological transfer from "bench to bedside", $\mathrm{BCl}$ must gain ease of use and robustness both in terms of measure and interface (signal processing and applications).

The RoBIK project (Robust Brain-computer Interface for virtual Keyboard) aimed at the development of a $\mathrm{BCl}$ system for communication that could be used on a daily basis by patients without the help of a trained team of researchers. In order to achieve such a challenging goal, a translational approach 
was chosen and developments were carefully framed with clinical specifications before the developments and clinical validation afterwards. To guide initial developments, clinicians first assessed patients' needs. The prototype developed consisted in a 14 felt-pad electrodes EEG headset sampling at $256 \mathrm{~Hz}$ by an electronic component capable of transmitting signals wirelessly. The application was a virtual keyboard generating a novel stimulation paradigm to elicit P300 Evoked Related Potentials (ERPs) for communication. Raw EEG signals were treated with OpenViBE opensource software running a specific signal processing chain including a novel Signal Quality Index (SQI) based on Riemannian geometry for artefacts rejection.

\section{Feasibility and evaluation of clinical needs}

Transferring BCls from computer sciences laboratories to patients' bedsides requires two important steps forward. First of all, the entire system (hardware and software) must comply with both patients and caretakers' needs. Second, applications tested with healthy volunteers needs to be evaluated with different populations of potential users in the very context of use, that is possibly flooded with many sort of noise: mechanical ventilation, various monitoring devices, and vibrating bed to cite few instances.

A questionnaire was written with occupational therapists specialized in providing effective assistive technology to patients presenting with a wide range of disabilities. The questionnaire was composed of three sections: general use of $\mathrm{BCl}, \mathrm{BCl}$ headset, and application. This survey $(n=40)$ highlighted the need for easy-to-install systems (installation time shorter than $15 \mathrm{~min}$ in $82 \%$ of responses). It also stressed the importance of mechanical comfort (selected as main priority by $72 \%$ of users and $60 \%$ of 
medical staff) with a daily use expected to be higher than 2 hours. In terms of application, communication was cited as a primary need by a large majority of patients followed by access to the Internet, emailing and demotic interfaces.

Evaluation of patients' needs also highlighted the presence of two distinct populations: patients with chronic disabilities such as neurodegenerative disorders and patients with acute conditions such as stroke and trauma patients. The chronic population is at home or in rehabilitation unit and sometimes already uses an assistive technology that must be over-performed to raise interest in $\mathrm{BCl}$ s. The acute population on the other hand, suddenly needs to fill the gap of communication, which could possibly be addressed by $\mathrm{BCls}$. In particular, quadriplegic patients who undergo mechanical ventilation are suddenly left speechless and can hardly benefit from other types of assistive technologies. Unfortunately, their environment (often Intensive Care Unit) is adverse for EEG measurement because of numerous uncontrolled sources of noise (electromagnetic, acoustic and mechanical). Moreover, acute conditions requires treatments that often includes different types of Central Nervous System (CNS) depressant, which can interfere with the BCI EEG features of interest.

Twelve quadriplegic patients admitted to ICU for whom verbal communication was compromised, were therefore enrolled in a feasibility study after giving informed consent. The aim of the clinical study is to assess the feasibility of a state-of-the-art $\mathrm{BCl}$ for communication [39]. Sixteen Ag-Cl disc electrodes were fitted to 10/20 standard locations and signals were sampled at $256 \mathrm{~Hz}$ with a Porti32 from TMSi (Twente, Netherlands). Digital signals and stimulations were handled by the open-source 
platform OpenViBE. Signals were filtered and transformed with the XDAWN spatial filter [55] prior to SVM voting classifier [56]. Results showed that $\mathrm{BCls}$ can be used in an Intensive Care Unit for restoration of communication despite, mechanical ventilation and use of CNS depressants.

\section{An EEG headset for Brain-Computer Interface}

The headset is composed of three main components:

1) 14 wet felt-pad electrodes,

2) The mechanical structure to hold the electrodes and the electronic module,

3) An electronic module to amplify, digitize and transmit EEG signal to the processing unit.

\section{General electronics architecture for miniaturized EEG amplifier}

Our goal was to develop a miniaturized electronics for EEG recording with a large number of electrodes (up to 32), while using as much as possible COTS (commercial-off-the-shelf) components. We used a MSP430 ultra-low power microcontroller to provide the control of the different modules, and a USB module from Silicon Labs. No component was identified as commercially available for EEG signal amplification and analog to digital conversion. Therefore, a dedicated Application Specific Integrated Component (ASIC) has been developed.

\section{Integrated Electronic: ASIC circuit for neural signal conversion (CINESIC)}

Interfacing electrodes using discrete electronics rapidly limits the number of channels, creating the need for highly integrated solutions to achieve sufficient spatial resolution. For this purpose, a 
dedicated ASIC CINESIC32 (CIrcuit for NEuronal SIgnal Conversion) has been developed with the two major constraints in mind: ultra low power consumption and patient's safety.

The ASIC filters, amplifies and digitizes the EEG data acquired from the electrodes. The architecture of CINESIC32 is shown in Figure 2. Each input channel is combined with an external capacitor (1.5nF) in order to suppress the risk of leaking current in a first default condition, which is essential for medical applications. The analogue channel is comprised of a fully differential low-noise amplifier, followed by a voltage gain amplifier and a programmable low-pass filter. The consumption of one analog channel is about $34 \mu \mathrm{A}$.

Digital peripherals such as configuration registers and a SPI (Serial Peripheral Interface) controller are also integrated on the chip. A special attention was paid on configurability to target different applications. A dedicated protocol was defined to address configuration registers. Consequently, the user can enable or disable each channel, configure the input switches in different modes, set the amplification stages in different gain (4 possible values: 1, 5, 200 and 1000) and set the frequency bandwidth $(B W 1=[0.5-300 \mathrm{~Hz}], \mathrm{BW}=[0.5-5000 \mathrm{~Hz}])$. For EEG applications, the channels will be configured to a $[0.5-300 \mathrm{~Hz}]$ bandwidth and a $60 \mathrm{~dB}$ voltage gain. Each analogue data is digitized through a 12-bit analog to digital converter (ADC). The nominal sampling frequency is $1 \mathrm{kHz}$ per channel. The CINESIC 32 chip was designed in complementary metal-oxide-semiconductor (CMOS) technology $(0.35 \mu \mathrm{m})$.

\section{Microcontroller module}

The MSP430F2618-EP from Texas Instruments was chosen for its ultra low power characteristics, its multiple communication interfaces. The MSP430 controls both the USB link and the data acquisition 
from the ASIC. A 3-axis accelerometer (ADXL345 from Analog Devices) is also connected to the microcontroller.

\section{The WIBEEM platform}

The WIBEEM (WIreless BCI EEG Electronics module) platform has been designed to take into account all the constraints of a wearable medical device: ultra-low power, miniaturization, safety and reliability and to be embedded on the headset. It is based on the general architecture presented above. The electronics module consumption at full data streaming conditions is around $13 \mathrm{~mA}$ at 3.3V. To guarantee 24 hours of continuous operation, the electronics operates on one high energy density 3.6V lithium battery. As shown in Figure 3, the WIBEEM module is made up of two printed circuit boards (PCB) linked by a board-to-board connector. The main components (ASIC and microcontroller) are placed on one side of the PCB (at the bottom) while the other PCB (at the top) contains the interface components (USB, LEDs, switch and connectors).

\section{RoBIK Graphical user interface}

The WIBEEM platform offers a Graphical User Interface (GUI) allowing rapid and easy setting of acquisition parameters like sampling frequency of the device and the gain of each electrode depending on the measured electrical activity. Through this GUI, EEG data are sent to the OpenViBE acquisition server through the fieldtrip buffer [57]. Furthermore, all data from the 32 channels can be saved and reloaded with the ROBIK GUI.

\section{A EEG headset}


In order to easily connect electrodes and get rid of the different steps of classical EEG recording (skin preparation, disc electrodes setup with gel, glue and tape) we designed an easy-to-use EEG headset. Electrodes are composed of a disposable felt pad (wet with saline solution) in contact with a SilverChloride electrode. Electrodes are mounted to a polyamide structure designed from a collection of 3D head models as seen on Figure 4. At each electrode site, a polyethurane handle controls the release of a spring that applies pressure on the scalp with the electrode offering a good contact and thereby a good signal quality. Ground and reference electrodes are disposable ECG electrodes to be located at each mastoïd. It takes less than 5 minutes to setup the whole system.

\section{A novel P300 Speller application}

\section{Signal Quality Index based on Riemannian geometry for artefact detection}

EEG artefacts can be divided in three families: biological, environmental and instrumental, with each family comprising several kind of artefacts. Depending on the amplitude and spatial distribution of the artefact the performance of a $\mathrm{P} 300 \mathrm{BCl}$ may be influenced very widely. We require to detect artefacts so as to minimize classification errors due to insufficient signal-to-noise ratio in the relevant EEG segment. Instead of trying to characterize every possible artefact, we have proposed to characterized the artefact-free state instead (Barachant et al., in press). The goal of the detection algorithm is to determine if a segment of EEG signal belongs to the artefact-free state or not. In order to do so, we work with the covariance matrices of the EEG segments. Covariance matrices belong to a special Riemann manifold wherein a Riemann metric can be used to define a distance between covariance matrices [58]. Using a few seconds of resting artefact-free data we estimate a region in the manifold using its barycentre and the variability of observations. More precisely, the region is 
defined as the mean \pm 2.5 standard deviations; when a new covariance matrix falls outside this region it is rejected. Since the Riemannian metric is non-linear, this region of interest corresponds to a "potato" in the Riemannian manifold, that is why in Barachant et al. (in press) the method is named "Riemann potato".

\section{Novel stimulation sequence for P300 generation}

In the original P300-speller paradigm symbols flash by rows and columns. Often detection errors arise because of the "adjacency-distraction" phenomenon $[59,60]$ non-target symbols in rows or columns adjacent to the target attract the user's attention when they flash, producing a P300 that makes the detection of the target P300 more difficult. To mitigate this effect we flash the symbols by random groups [61]. Not only the "adjacency-distraction" effect is mitigated, we also obtain that the pattern of flashing becomes totally unpredictable, which is expected to sustain the attention of the user. Noteworthy, random-group flashing allows arbitrary positioning of the symbols on the screen (no more need to arrange symbols on a grid), which greatly expand the usability of the P300 paradigm. This feature has been exploited in our user interface (see below).

Usually, the stimulus interval (the flashing time) and the inter-stimulus interval (ISI: the time between two flashes) are kept constant. The periodic flashing is annoying and tiring because the visual cortex is driven to oscillate at the flashing frequency, which is usually far away from the natural talamocortical loop oscillation of this region, which is in the alpha range $(8-12 \mathrm{~Hz})$. Furthermore, the periodic flashing makes the flashing pattern predictable and boring. To eliminate all these effects we may use random ISI drawn from a random exponential distribution. The exponential distribution (also called 
"waiting-time" distribution) with parameter $\lambda$ and both population mean and population standard deviation $=1 / \lambda$ is the distribution of the time passing in between two events of random series following a Poisson process with the same parameter $\lambda$, population mean $=\lambda$ and population standard deviation $=\sqrt{ } \lambda$. This is a process in which events occur continuously and independently at a constant average rate. For example, it is the natural distribution for modelling time between system failures, telephone calls, customer arrivals, accidents at a street intersection, etc.

\section{Brainmium: A P300-based web browser}

Brainmium is a standalone web browser that enables navigating within a web page using the P300 Speller paradigm. More precisely, Brainmium is a software framework that allows the execution and development of P300-based web applications, which we refer as "Brainmium apps". A Brainmium app is defined such as a standard web page, in HTML and JavaScript, but enhanced, either statically or at runtime, by some Brainmium-specific tags. Brainmium engine infers the HTML elements of interest from these tags. In general, these elements correspond to the HTML links or images contained within a web page. In order to select a given element, Brainmium makes use of a state-ofthe-art P300 spelling paradigm [61].

A few studies made in the literature have proposed to address the needs of browsing the World Wide Web (WWW) using a P300 paradigm [62-65]. In most of these proposals, however, the $\mathrm{BCl}$ system is built on top of a regular web browser coupled to a separate P300 Speller application (i.e.

run into separate windows). This speller matrix usually consists in the largest possible set of symbols necessary to navigate between links or to use the browser native functions. The navigation itself 
could therefore become increasingly difficult depending on the web content, resulting in a slow throughput and finally users disinterest in the tool [62]. Visual complexity of web contents [66] is one of the reason to explain the poor performance of these paradigms.

With Brainmium, we adopted a different approach to leverage this complexity. In fact, our main focus is not really tied to browsing Internet resources, even though this is possible. Instead, we rely upon web technologies to facilitate the prototyping and development of P300-based applications. Our system is in fact developed in a way reminiscent to that of modern mobile systems [67]. In other words, Brainmium acts as an application container that proposes a subset of the facilities usually found in mobile systems, such as an application dashboard, a configurable virtual keyboard. A development toolkit will be proposed to achieve this goal. It is also important to note that unlike a conventional P300 Speller implementation, the symbols are not necessarily arranged into a square matrix. Flashing items into random groups makes this strategy possible and efficient [61].

As detailed in Figure 5, Brainmium relies upon the OpenVibe platform [43] to acquire EEG data and treat them in order to detect P300 evoked response potentials. In this regard, the main task of Brainmium is to take care of displaying frame-accurate visual stimuli and to communicate the respective timestamps to OpenViBE. Brainmium and OpenViBE are connected through a shared memory module, which offers a very fast and almost negligible transfer time. Once a P300 event is detected, OpenViBE uses a Virtual-Reality Peripheral Network (VRPN) tunnel [43] to send the data back to Brainmium, which performs target selection. 
Brainmium is developed in C\# and its rendering engine is built on top of Microsoft XNA / DirectX to obtain an accurate timing for P300 flashes. For the experiments, we have considered a fixed framerate set to 120 Frame-Per-Second.

\section{Conclusion}

The future of $\mathrm{BCl}$ s as an assistive technology depends on the good understanding of patients' and caretakers' needs. In particular, the distinction between different subsets of patients (such as chronic versus acute) allows the identification of adequate paradigms and applications. Our clinical survey $(n=40)$ placed communication as a primary need and revealed the importance of a short setup time ( $t<15 \mathrm{~min}$ ) for the whole system. The prototype developed during the RoBIK project was therefore an easy-to-set-up EEG headset with fourteen wet electrodes, allowing the control of a web based P300 spelling interface. The EEG headset was connected to a high quality electronic component designed with low noise and high input impedance. The application embedded novel signal processing and classification algorithms based on Riemannian geometry offering unequal performance together with an automated rejection of artefacts. The performance of the whole system is currently being investigated in a multicentre randomized control trial comparing its performance to the one of traditional scanning spelling systems. 


\section{References}

1. Vidal JJ: Toward direct brain-computer communication. Annual review of biophysics and bioengineering 1973, 2:157-180.

2. Wolpaw JR, Birbaumer N, Heetderks WJ, McFarland DJ, Peckham PH, Schalk G, Donchin E, Quatrano LA, Robinson CJ, Vaughan TM: Brain-computer interface technology: a review of the first international meeting. IEEE transactions on rehabilitation engineering : a publication of the IEEE Engineering in Medicine and Biology Society 2000, 8(2):164-173.

3. Chan E: Food and Drug Administration and the Future of Brain-Computer Interface: Adapting FDA Device Law to the Challenges of Human-Machine Enhancement, The. $J$ Marshall J Computer \& Info L 2007, 25:117.

4. Marin C, Fernandez E: Biocompatibility of intracortical microelectrodes: current status and future prospects. Frontiers in neuroengineering 2010, 3:8.

5. Wilson JA, Felton EA, Garell PC, Schalk G, Williams JC: ECoG factors underlying multimodal control of a brain-computer interface. IEEE transactions on neural systems and rehabilitation engineering : a publication of the IEEE Engineering in Medicine and Biology Society 2006, 14(2):246-250.

6. Leuthardt EC, Schalk G, Wolpaw JR, Ojemann JG, Moran DW: A brain-computer interface using electrocorticographic signals in humans. Journal of neural engineering 2004, 1(2):63.

7. Sitaram R, Caria A, Veit R, Gaber T, Rota G, Kuebler A, Birbaumer N: FMRI brain-computer interface: a tool for neuroscientific research and treatment. Computational intelligence and neuroscience 2007:25487.

8. Weiskopf N, Mathiak K, Bock SW, Scharnowski F, Veit R, Grodd W, Goebel R, Birbaumer N: Principles of a brain-computer interface $(\mathrm{BCl})$ based on real-time functional magnetic resonance imaging (fMRI). IEEE transactions on bio-medical engineering 2004, 51(6):966970.

9. Weiskopf N, Mathiak K, Bock SW, Scharnowski F, Veit R, Grodd W, Goebel R, Birbaumer N: Principles of a brain-computer interface $(\mathrm{BCl})$ based on real-time functional magnetic resonance imaging (fMRI). Biomedical Engineering, IEEE Transactions on 2004, 51(6):966970.

10. Mellinger J, Schalk G, Braun C, PreissI H, Rosenstiel W, Birbaumer N, Kubler A: An MEGbased brain-computer interface (BCl). Neurolmage 2007, 36(3):581-593.

11. Kauhanen L, Nykopp T, Lehtonen J, Jylanki P, Heikkonen J, Rantanen P, Alaranta H, Sams $M$ : EEG and MEG brain-computer interface for tetraplegic patients. IEEE transactions on neural systems and rehabilitation engineering : a publication of the IEEE Engineering in Medicine and Biology Society 2006, 14(2):190-193.

12. Sitaram R, Zhang H, Guan C, Thulasidas M, Hoshi Y, Ishikawa A, Shimizu K, Birbaumer N: Temporal classification of multichannel near-infrared spectroscopy signals of motor imagery for developing a brain-computer interface. Neurolmage 2007, 34(4):1416-1427.

13. Coyle S, Ward T, Markham C, McDarby G: On the suitability of near-infrared (NIR) systems for next-generation brain-computer interfaces. Physiological measurement 2004, 25(4):815-822.

14. Wolpaw JR, McFarland DJ, Neat GW, Forneris CA: An EEG-based brain-computer interface for cursor control. Electroencephalography and clinical neurophysiology 1991, 78(3):252-259. 
15. Birbaumer $\mathrm{N}$ : Breaking the silence: brain-computer interfaces $(\mathrm{BCl})$ for communication and motor control. Psychophysiology 2006, 43(6):517-532.

16. Wolpaw JR: Brain-computer interfaces (BCls) for communication and control: a minireview. Supplements to Clinical neurophysiology 2004, 57:607-613.

17. Wolpaw JR, Birbaumer N, McFarland DJ, Pfurtscheller G, Vaughan TM: Brain-computer interfaces for communication and control. Clinical neurophysiology : official journal of the International Federation of Clinical Neurophysiology 2002, 113(6):767-791.

18. Sellers EW, Vaughan TM, Wolpaw JR: A brain-computer interface for long-term independent home use. Amyotrophic Lateral Sclerosis 2010, 11(5):449-455.

19. Grozea C, Voinescu CD, Fazli S: Bristle-sensors--low-cost flexible passive dry EEG electrodes for neurofeedback and $\mathrm{BCl}$ applications. Journal of neural engineering 2011 , 8(2):025008.

20. Wang Y, Gao X, Hong B, Jia C, Gao S: Brain-computer interfaces based on visual evoked potentials. IEEE engineering in medicine and biology magazine : the quarterly magazine of the Engineering in Medicine \& Biology Society 2008, 27(5):64-71.

21. Sellers E, Turner P, Sarnacki W, McManus T, Vaughan T, Matthews R: A novel dry electrode for brain-computer interface. Human-Computer Interaction Novel Interaction Methods and Techniques 2009:623-631.

22. Popescu F, Fazli S, Badower Y, Blankertz B, Müller KR: Single trial classification of motor imagination using 6 dry EEG electrodes. PloS one 2007, 2(7):e637.

23. Teplan M: Fundamentals of EEG measurement. Measurement science review 2002, 2(2):111.

24. Hornecker A: Dear Customers, Dear Friends of Brain Products. Brain : a journal of neurology 2009, 33.

25. Renard Y: IN THE FOCUS. Brain : a journal of neurology 2010, 37.

26. Millan JD, Rupp R, Muller-Putz GR, Murray-Smith R, Giugliemma C, Tangermann M, Vidaurre C, Cincotti F, Kubler A, Leeb R et al: Combining Brain-Computer Interfaces and Assistive Technologies: State-of-the-Art and Challenges. Frontiers in neuroscience 2010, 4.

27. Nijholt A, Bos DPO, Reuderink B: Turning shortcomings into challenges: Brain-computer interfaces for games. Entertainment Computing 2009, 1(2):85-94.

28. Emotiv E: Software Development Kit. In.; 2010.

29. Stytsenko K, Jablonskis E, Prahm C: Evaluation of consumer EEG device emotiv epoc. In: MEi: CogSci Conference 2011, Ljubljana: 2011; 2011.

30. Duvinage M, Castermans T, Dutoit T, Petieau M, Hoellinger T, De Saedeleer C, Seetharaman K, Cheron G: A P300-based Quantitative Comparison between the Emotiv Epoc Headset and a Medical EEG Device. In: Biomedical Engineering/765: Telehealth/766: Assistive Technologies: 2012: ACTA Press; 2012.

31. Liu Y, Jiang X, Cao T, Wan F, Mak PU, Mak PI, Vai MI: Implementation of SSVEP based BCI with Emotiv EPOC. In: Virtual Environments Human-Computer Interfaces and Measurement Systems (VECIMS), 2012 IEEE International Conference on: 2012: IEEE; 2012: 34-37.

32. Bai O, Lin P, Vorbach S, Floeter MK, Hattori N, Hallett M: A high performance sensorimotor beta rhythm-based brain-computer interface associated with human natural motor behavior. Journal of neural engineering 2007, 5(1):24, 1741-2552.

33. Birbaumer $\mathrm{N}$ : Breaking the silence: brain-computer interfaces $(\mathrm{BCl})$ for communication and motor control. Psychophysiology 2006, 43(6):517-532 1469-8986.

34. Pfurtscheller G, Brunner C, Schlögl A, Lopes SFH: Mu rhythm (de) synchronization and EEG single-trial classification of different motor imagery tasks. Neurolmage 2006, 31(1):153 1053-8119. 
35. Allison B, Luth T, Valbuena D, Teymourian A, Volosyak I, Graser A: BCI Demographics: How many (and what kinds of) people can use an SSVEP BCI? Neural Systems and Rehabilitation Engineering, IEEE Transactions on 2010, 18(2):107-116, 1534-4320.

36. Pfurtscheller G, Solis-Escalante T, Ortner R, Linortner P, Muller-Putz G: Self-paced operation of an SSVEP-Based orthosis with and without an imagery-based "brain switch:" a feasibility study towards a hybrid BCl. Neural Systems and Rehabilitation Engineering, IEEE Transactions on 2010, 18(4):409-414, 1534-4320.

37. Picton TW: The P300 wave of the human event-related potential. Journal of clinical neurophysiology : official publication of the American Electroencephalographic Society 1992, 9(4):456-479.

38. Kaper M, Meinicke P, Grossekathoefer U, Lingner T, Ritter H: BCl competition 2003-data set llb: Support vector machines for the P300 speller paradigm. Biomedical Engineering, IEEE Transactions on 2004, 51(6):1073-1076, 0018-9294.

39. Krusienski DJ, Sellers EW, McFarland DJ, Vaughan TM, Wolpaw JR: Toward enhanced P300 speller performance. Journal of neuroscience methods 2008, 167(1):15.

40. Nijboer F, Furdea A, Gunst I, Mellinger J, McFarland DJ, Birbaumer N, Kübler A: An auditory brain-computer interface (BCl). Journal of neuroscience methods 2008, 167(1):43-50, 0165-0270.

41. Bashashati A, Fatourechi M, Ward RK, Birch GE: A survey of signal processing algorithms in brain-computer interfaces based on electrical brain signals. Journal of neural engineering 2007, 4(2):R32, 1741-2552.

42. Lotte $\mathrm{F}$, Congedo $\mathrm{M}$, Lécuyer $\mathrm{A}$, Lamarche $\mathrm{F}$, Arnaldi B: A review of classification algorithms for EEG-based brain-computer interfaces. Journal of neural engineering 2007, 4.

43. Renard Y, Lotte F, Gibert G, Congedo M, Maby E, Delannoy V, Bertrand O, Lécuyer A: OpenViBE: an open-source software platform to design, test, and use brain-computer interfaces in real and virtual environments. Presence: teleoperators and virtual environments 2010, 19(1):35-53, 1054-7460.

44. Schalk G, McFarland DJ, Hinterberger T, Birbaumer N, Wolpaw JR: BCl2000: a generalpurpose brain-computer interface $(\mathrm{BCl})$ system. Biomedical Engineering, IEEE Transactions on 2004, 51(6):1034-1043, 0018-9294.

45. Wolpaw JR, Birbaumer N, McFarland DJ, Pfurtscheller G, Vaughan TM: Brain-computer interfaces for communication and control. Clinical neurophysiology 2002, 113(6):767-791 \%@1388-2457.

46. Philips J, del R Millan J, Vanacker G, Lew E, Galán F, Ferrez PW, Van Brussel H, Nuttin M: Adaptive shared control of a brain-actuated simulated wheelchair: IEEE; 2007.

47. Rebsamen B, Guan C, Zhang H, Wang C, Teo C, Ang MH, Burdet E: A brain controlled wheelchair to navigate in familiar environments. Neural Systems and Rehabilitation Engineering, IEEE Transactions on 2010, 18(6):590-598, 1534-4320.

48. Bell CJ, Shenoy P, Chalodhorn R, Rao RPN: Control of a humanoid robot by a noninvasive brain-computer interface in humans. Journal of neural engineering 2008, 5(2):214, 1741-2552.

49. Cincotti F, Mattia D, Aloise F, Bufalari S, Schalk G, Oriolo G, Cherubini A, Marciani MG, Babiloni F: Non-invasive brain-computer interface system: towards its application as assistive technology. Brain research bulletin 2008, 75(6):796-803, 0361-9230.

50. Sellers EW, Donchin E: A P300-based brain-computer interface: initial tests by ALS patients. Clinical neurophysiology 2006, 117(3):538-548, 1388-2457.

51. Kübler A, Nijboer F, Mellinger J, Vaughan TM, Pawelzik H, Schalk G, McFarland D, Birbaumer $\mathrm{N}$, Wolpaw JR: Patients with ALS can use sensorimotor rhythms to operate a braincomputer interface. Neurology 2005, 64(10):1775-1777, 0028-3878. 
52. Leuthardt EC, Schalk G, Wolpaw JR, Ojemann JG, Moran DW: A brain-computer interface using electrocorticographic signals in humans. Journal of neural engineering 2004, 1(2):63\%@1741-2552.

53. Piccione F, Giorgi F, Tonin P, Priftis K, Giove S, Silvoni S, Palmas G, Beverina F: P300based brain computer interface: reliability and performance in healthy and paralysed participants. Clinical neurophysiology 2006, 117(3):531-537, 1388-2457.

54. Wolpaw JR, McFarland DJ, Vaughan TM: Brain-computer interface research at the Wadsworth Center. Rehabilitation Engineering, IEEE Transactions on 2000, 8(2):222-226, 1063-6528.

55. Rivet B, Souloumiac A, Attina V, Gibert G: XDAWN algorithm to enhance evoked potentials: application to brain-computer interface. Biomedical Engineering, IEEE Transactions on 2009, 56(8):2035-2043, 0018-9294.

56. Rakotomamonjy A, Guigue V: BCl competition III: dataset II-ensemble of SVMs for BCI P300 speller. Biomedical Engineering, IEEE Transactions on 2008, 55(3):1147-1154, 00189294.

57. Oostenveld R, Fries P, Maris E, Schoffelen JM: FieldTrip: Open source software for advanced analysis of MEG, EEG, and invasive electrophysiological data. Computational intelligence and neuroscience 2011, 2011:156869.

58. Barachant A, Bonnet S, Congedo M, Jutten C: Multiclass brain-computer interface classification by Riemannian geometry. IEEE transactions on bio-medical engineering 2012, 59(4):920-928.

59. Jin SH, Lin P, Hallett M: Abnormal reorganization of functional cortical small-world networks in focal hand dystonia. PloS one 2011, 6(12):e28682.

60. Townsend G, LaPallo BK, Boulay CB, Krusienski DJ, Frye GE, Hauser CK, Schwartz NE, Vaughan TM, Wolpaw JR, Sellers EW: A novel P300-based brain-computer interface stimulus presentation paradigm: moving beyond rows and columns. Clinical neurophysiology : official journal of the International Federation of Clinical Neurophysiology 2010, 121(7):1109-1120.

61. Congedo M, Goyat M, Tarrin N, Ionescu G, Varnet L, Rivet B, Phlypo R, Jrad N, Acquadro M, Jutten C: 'Brain Invaders': a prototype of an open-source P300-based video game working with the OpenViBE platform. In: Proceedings of the 5th International BrainComputer Interface Conference 2011: 2011; 2011: 280-283.

62. Mugler EM, Ruf CA, Halder S, Bensch M, Kubler A: Design and implementation of a P300based brain-computer interface for controlling an internet browser. IEEE transactions on neural systems and rehabilitation engineering : a publication of the IEEE Engineering in Medicine and Biology Society 2010, 18(6):599-609.

63. Muglerab E, Benschc M, Haldera S, Rosenstielc W, Bogdancd M, Birbaumerae N, Kübleraf A: Control of an internet browser using the P300 event-related potential. 2008.

64. Bensch M, Karim AA, Mellinger J, Hinterberger T, Tangermann M, Bogdan M, Rosenstiel W, Birbaumer N: Nessi: an EEG-controlled web browser for severely paralyzed patients. Computational intelligence and neuroscience 2007, 2007.

65. Karim AA, Hinterberger T, Richter J, Mellinger J, Neumann N, Flor H, Kübler A, Birbaumer N: Neural internet: Web surfing with brain potentials for the completely paralyzed. Neurorehabilitation and Neural Repair 2006, 20(4):508-515.

66. Harper S, Jay C, Michailidou E, Quan H: Analysing the Visual Complexity of Web Pages Using Document Structure.

67. Rogers R, Lombardo J, Mednieks Z, Meike B: Android application development: Programming with the Google SDK: O'Reilly Media, Inc.; 2009. 


\section{Figures}

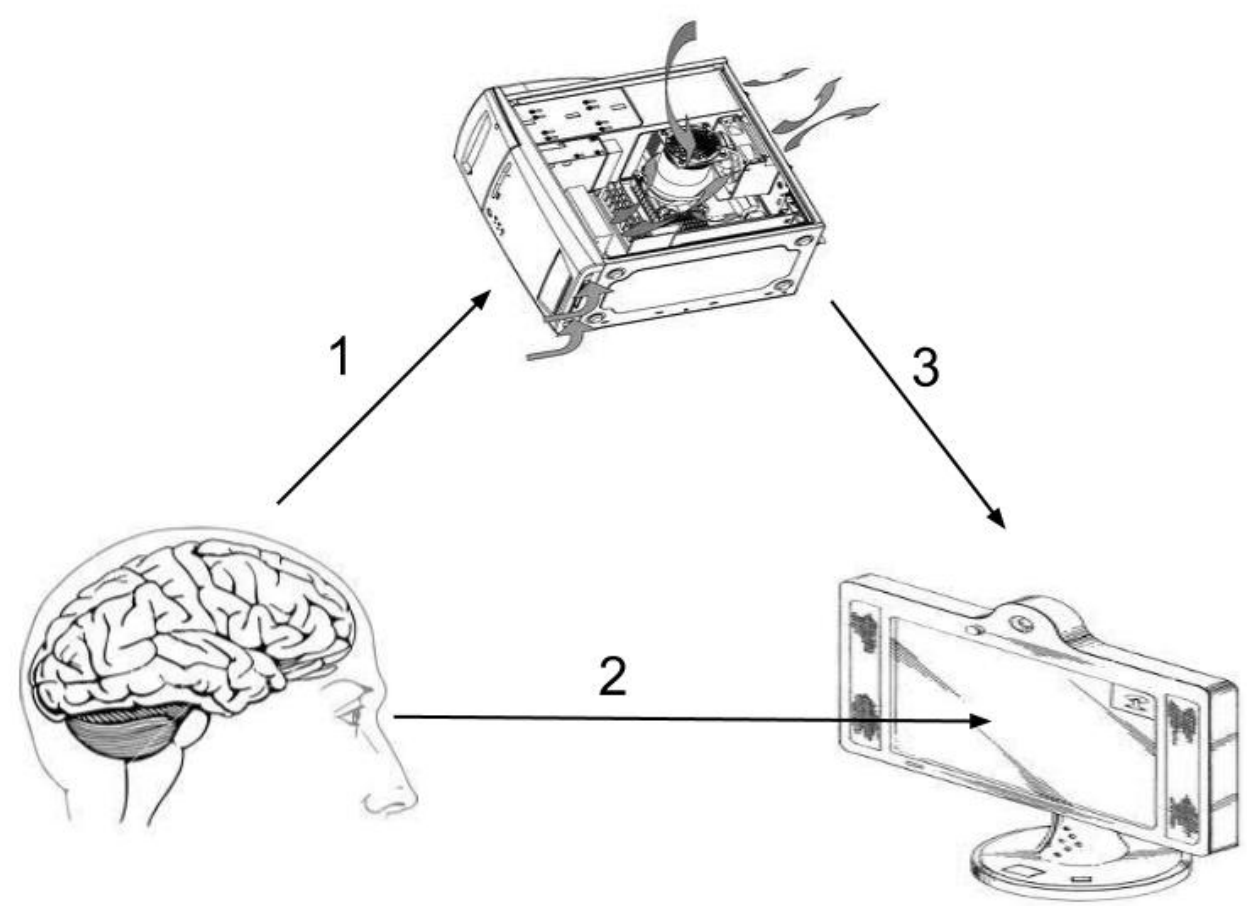

Figure 1: Principle of a Brain Computer Interface (BCl) based on Evoked Related Potentials (ERPs): EEG signals are recorded (1) while a computer generates a series of stimulations that generates EEG features (2) 


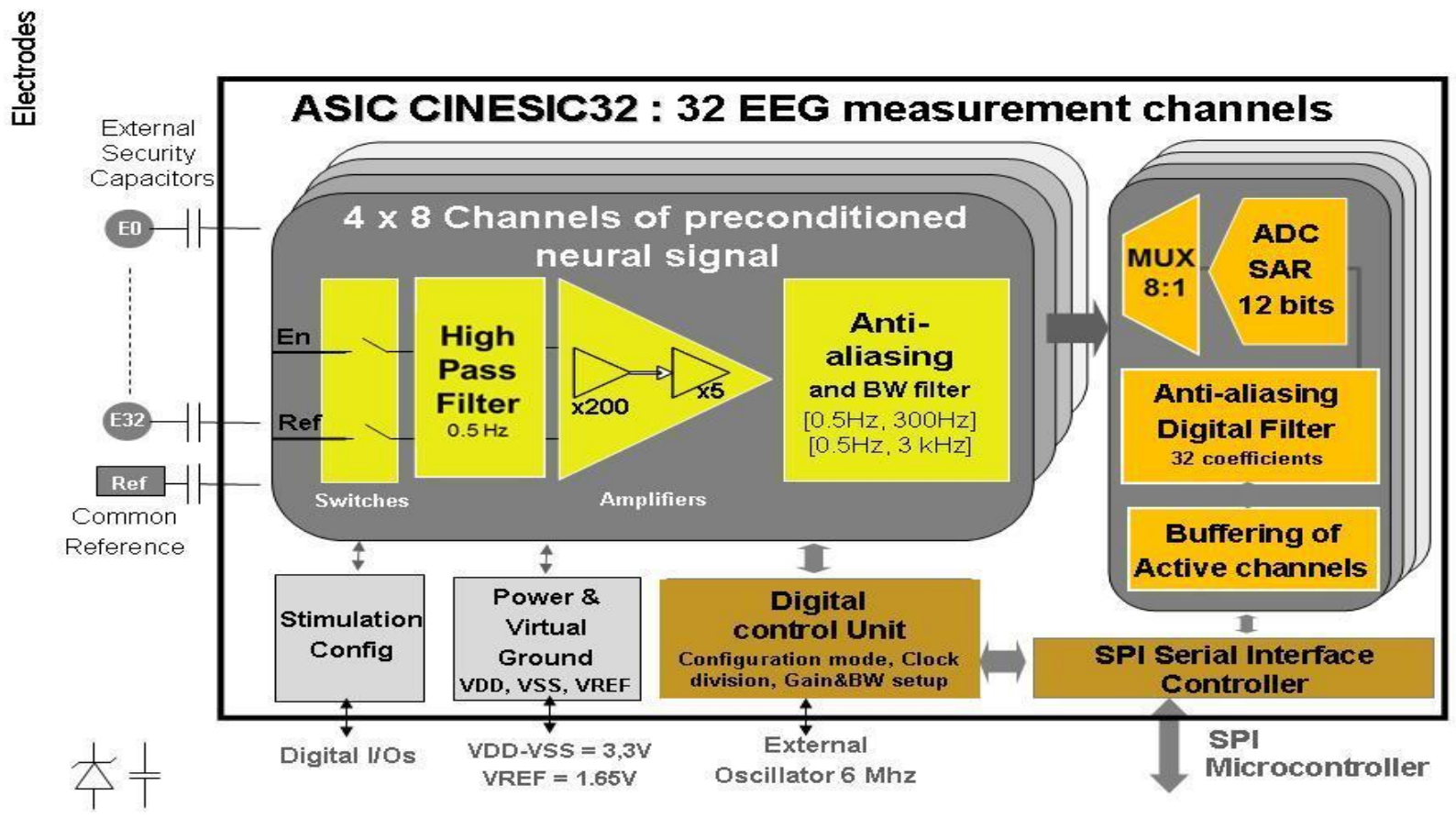

Figure 2: Architecture of the circuit for neural signal conversion (CINESIC) Application Specific Integrated Component (ASIC).

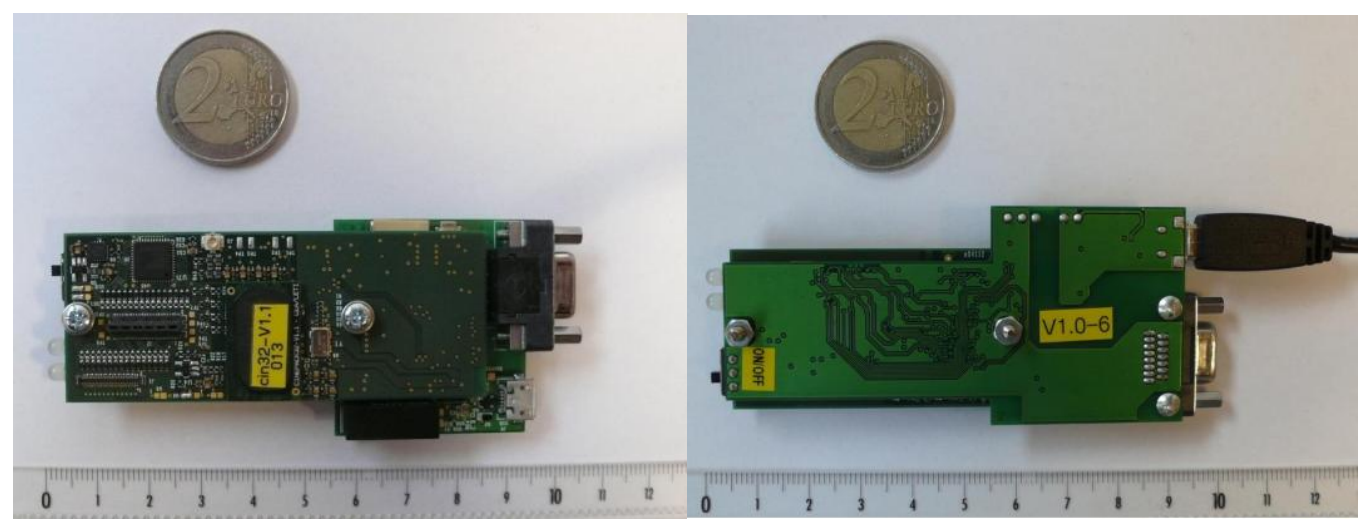

Figure 3: Pictures of the WIreless BCI EEG Electronics Module (WIBEEM): bottom and top de la printed circuit board (PCB). 


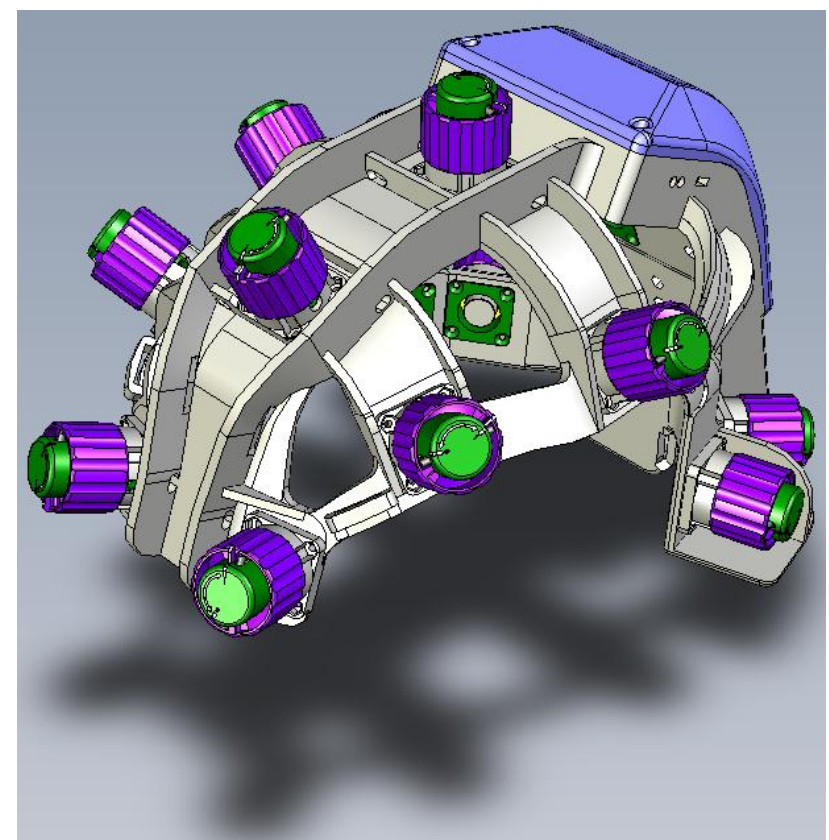

Figure 4: 3D view of the RoBIK headset showing the electrode handle (purple) and the box (blue) containing the electronics and battery.

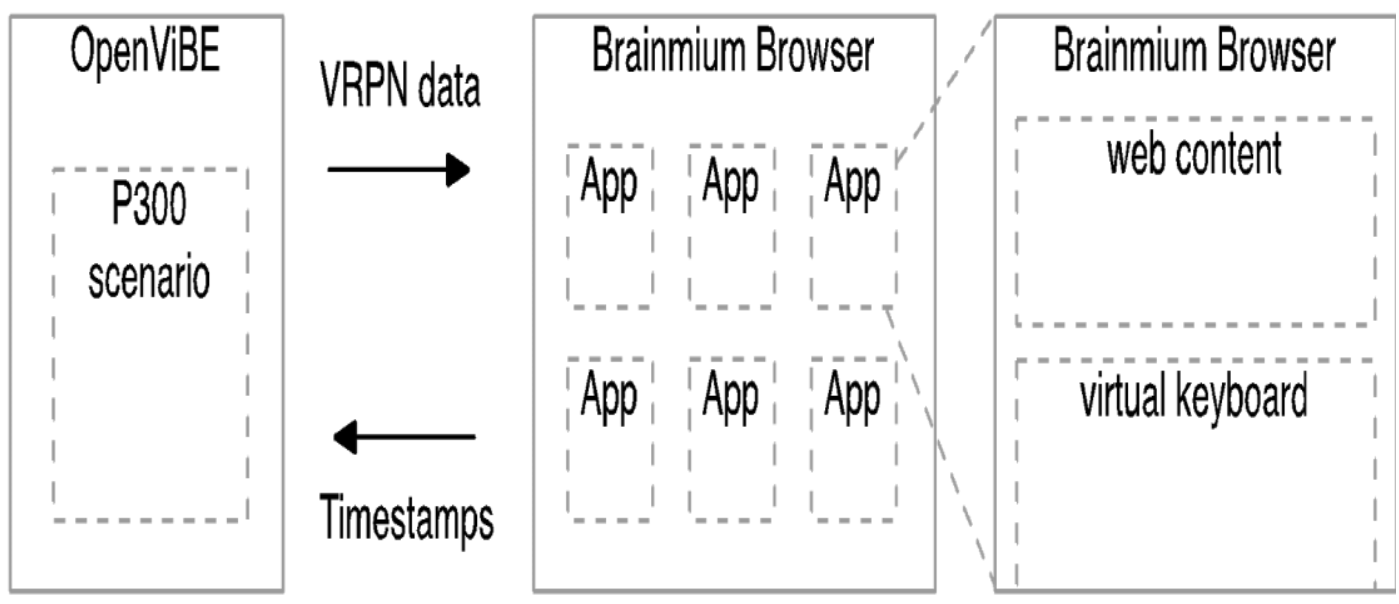

Figure 5: Brainmium architecture 